\title{
Beckenverletzungen - externe Fixation und Komplikation
}

\author{
Franz Maurer
}

\section{Zusammenfassung}

Fixateur externe und Beckenzwinge sind bewährte Hilfsmittel zur notfallmäßigen Stabilisierung von instabilen Beckenringverletzungen. Während der Fixateur externe vor allem für die ventrale Instabilität gedacht ist, bleibt die hintere Beckenringinstabilität eine Domäne der Beckenzwinge. Trotz der schnellen und einfachen Anwendbarkeit beider Stabilisierungsverfahren müssen Komplikations- möglichkeiten bedacht werden. Die Risiken ergeben sich zum Einen aus der Fehlplatzierung von Verankerungsschrauben mit Verletzung benachbarter Organe, zum Anderen aber vor allem auch aus lokalen Weichteilkomplikationen bei längeren Liegezeiten. Deswegen sollten beide externe Fixationsmöglichkeiten nur eine vorübergehende Maßnahme bis zur definitiven internen Stabilisierung des Beckenrings darstellen.

\section{Einleitung}

Instabile Beckenverletzungen höherer Schweregrade (B- und C-Verletzungen) gehen nicht nur mit Schmerzen, sondern auch immer wieder mit kreislaufwirksamen inneren Blutungen einher (Pohlemann). Diese Erfahrung hat dazu geführt, dass bereits vor der Klinikaufnahme im Rahmen der notärztlichen Erstversorgung Stabilisierungsmaßnahmen wie pneumatischer Beckengürtel, Antischock-Hose und Vakuummatratze zur Anwendung kommen. Nur noch historisch interessant sind in der klinischen Behandlung Stabilisierungsversuche wie die sogenannte Becken- oder Rauchfußschwebe. Selbst die Extensionsbehandlung kommt nur noch in wenigen Sonderfällen als Therapie infrage.

Durch die unkomplizierte Anwendbarkeit sowie hohe Effektivität haben Fixateur externe und Beckenzwinge als äußere Stabilisierungsverfahren einen festen Platz in der Notfallbehandlung der Be-

OP-JOURNAL 2003; 19: 128-131

(C) Georg Thieme Verlag Stuttgart . New York ckenringverletzungen. Selten wird eine externe Stabilisierung mit dem Fixateur externe als Ergänzung zur inneren Osteosynthese oder auch als alleinige Stabilisierungsmaßnahme in der Ausbehandlung einer Beckenringverletzung eingesetzt.

\section{Hauptteil}

\section{Hinterer Beckenring/Beckenzwinge}

Bereits Richter hatte in Freiburg eine einfache Beckenzwinge zur Stabilisierung des hinteren Beckenrings entwickelt. Noch heute basiert die modernere Ausführung der AO-Beckenzwinge auf dem Prinzip der Tischlerzwinge.

Die klassische Indikation für die Anwendung der Beckenzwinge ist die Beckenzerreißung mit hinterer Instabilität und kreislaufwirksamer Blutung aus dem retroperitonealen Venenplexus oder freiliegenden spongiösen Frakturflächen. In dieser Situation ist die Blutstillung durch Selbsttamponade erschwert, weil das Becken als stabiles Kompartiment nicht mehr besteht. Deswegen wird versucht, durch äußere Kompression und Stabilisierung mit der Beckenzwinge die Blutstillung zu unterstützen (Ruedi).
Entscheidend für die Wirksamkeit der Beckenzwinge ist die exakte Platzierung der Verankerungsschrauben in beiden Darmbeinaußenflächen.

Da das Einbringen dieser Verankerungsschrauben allein anhand der Orientierung mittels äußerer entweder tastbarer oder sichtbarer Landmarken möglich ist, kann die Beckenzwinge bereits im Schockraum oder auch auf der Intensivstation ohne Durchleuchtungskontrolle angelegt werden. Der Patient befindet für die Anlage der Beckenzwinge in Rückenlagerung, die Inzision für das Einführen der Verankerungsschrauben liegt auf der Schnittstelle einer Verbindungslinie Spina iliaca anterior superior Spina iliaca posterior superior und der Femurlängsachse (Abb.1). Bei vertikaler Verschiebung kann noch vor Ausübung der Kompression durch Zug am Bein eine Grobreposition erfolgen.

Nach Einbringen der Dorne der Verankerungsschrauben in die Darmbeinaußenfläche werden die beiden Arme der Beckenzwinge auf der Verbindungsschiene

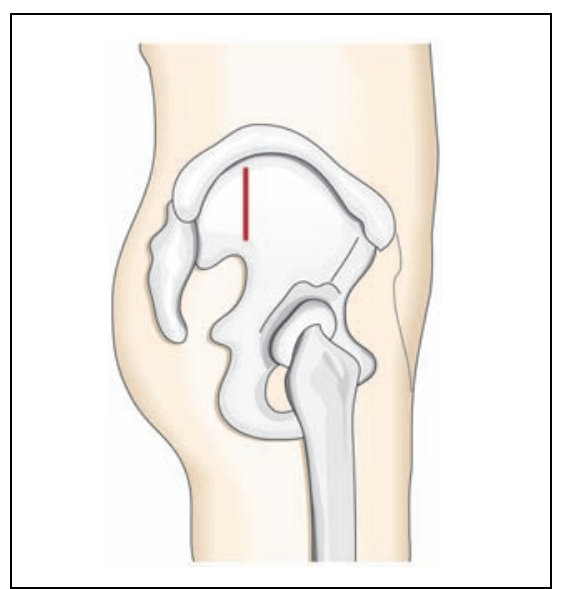

Abb.1 Optimaler Insertionspunkt der Verankerungsschrauben der Beckenzwinge an der Schnittstelle der Verbindung Spina iliaca anterior superior und - posterior superior sowie der Femurlängsachse. 


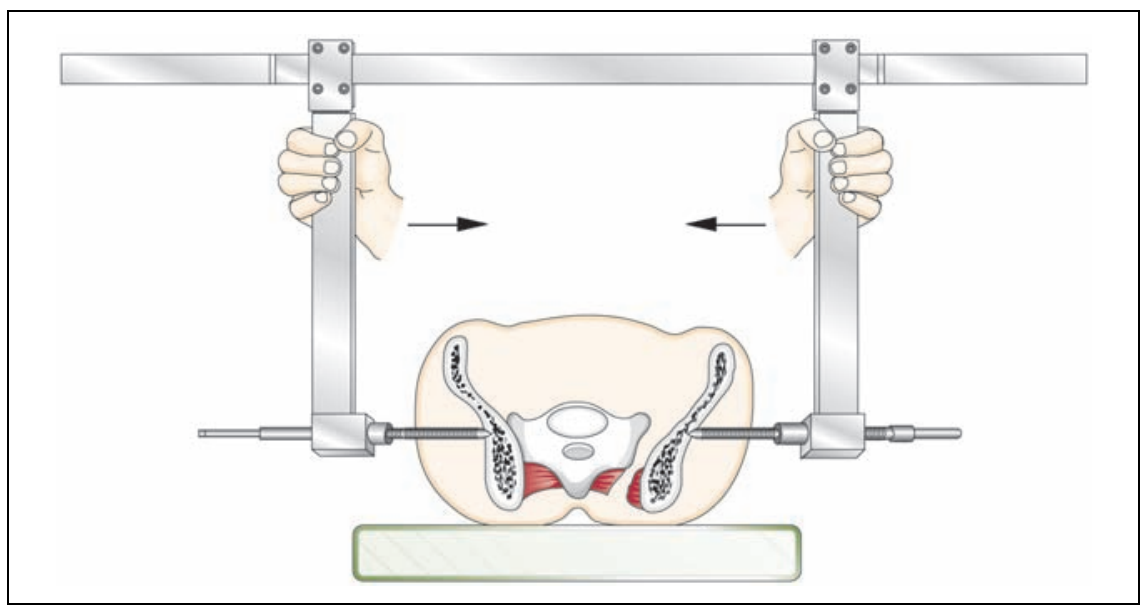

Abb. 2 Reposition und Kompression einer instabilen SI-Fuge durch Zusammenführen der beiden Arme der Beckenzwinge auf der Verbindungsschiene.

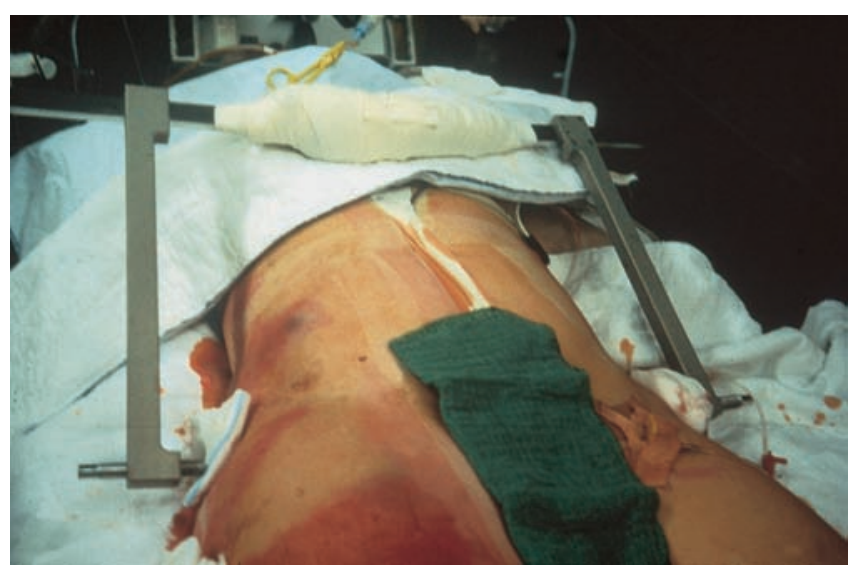

Abb. 3 Beispiel für die klinische Anwendung der Beckenzwinge beim Schwerverletzten. Freier Zugang zur Laparotomiewunde durch Abklappen der Beckenzwinge nach kranial.

auf den kleinstmöglichen Abstand gebracht, danach kann durch Vordrehen der Verankerungsschrauben noch ein weiterer Kompressionseffekt ausgeübt werden (Abb.2).

Eine liegende Beckenzwinge behindert in der Regel den Zugang zum Abdomen oder auch eine computertomographische Untersuchung nicht, da der Bügel ohne Verlust der Haltekraft nach kranial oder kaudal abgeklappt werden kann (Abb.3).

Risiken:

Die Komplikationsmöglichkeiten ergeben sich aus der Fehllage der Verankerungsschrauben, auch sind lokale Weichteilprobleme zu beachten:

1. Perforation des Darmbeins

2. Stabilitätsverlust

3. Verletzung des glutaealen GefäßNervenbündels

4. Verletzung des $N$. ischiadicus

5. Kompression von Sakralnerven
6. Verkippen des Darmbeins

7. Weichteilprobleme der Eintrittsstelle

Sofern die Landmarken zur Orientierung schlecht sicht- oder tastbar sind, kann die Anbringung der Beckenzwinge erschwert sein. Es besteht dann ein erhöhtes Risiko für eine Fehlplatzierung der Verankerungsschrauben und sich daraus ergebende Komplikationsmöglichkeiten. Bei Insertion im Bereich des dünnen Anteils des Os iliums kommt es zur Perforation in Richtung der Beckenorgane mit dem Risiko einer Verletzung verschiedener Strukturen, abgesehen von der mangelnden Festigkeit der Montage in solch einem Fall.

Je näher die Verankerungsschrauben in Richtung Foramen ischiadicum eingebracht werden, desto größer wird das Risiko einer Verletzung des glutaealen Gefäß-Nervenbündels. Die gravierenden Folgen eines bleibenden Schadens der Glutaealnerven für das Gehen (Glutaealinsuffizienz) und der Gefäße (Arteria

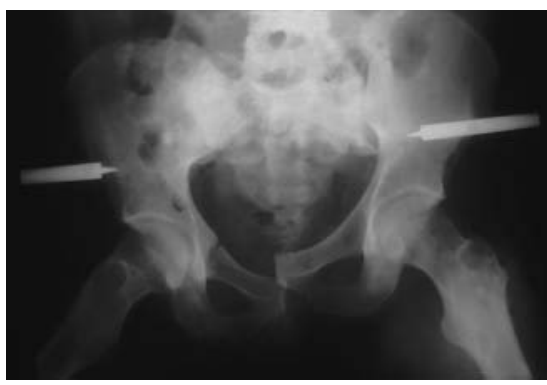

Abb.4 Dislokation des vorderen Beckenrings durch fehlende Reposition der hinteren Beckenringanteile und Kompression mittels Beckenzwinge.

glutaea ist Endarterie) sind hinreichend bekannt. Mit dem Abweichen der Schrauben ins Foramen ischiadicum selbst gerät auch der Nervus ischadicus in Gefahr.

Bei Insertion der Verankerungsschrauben außerhalb des biomechanisch günstigen iliosakralen Fensters kann eine Hebelwirkung auf das Os ilium entstehen und eine Rotationsbewegung mit Verkippen des Darmbeins bei Ausübung der Kompression resultieren (Abb.4).

Die zu starke Kompression mit gleichzeitigem Vorliegen einer Sakrumfraktur durch die Nervenaustrittslöcher hindurch können Teile des Plexus sacralis in Mitleidenschaft gezogen werden. Die Nerven werden in diesem Fall im Foramen komprimiert und dadurch geschädigt.

Nicht vergessen werden soll auch das Problem der Weichteilirritation an der Eintrittsstelle der Verankerungsschrauben. Bei voluminösem Weichteilmantel und ungenügendem Spielraum durch eine ausreichend große Inzision können lokale Nekrosen und eine chronische Weichteilentzündung resultieren, wobei im Endeffekt immer die Infektion entlang der Schraube in die Tiefe droht. Allerdings ist das Infektionsrisiko bei entsprechender sorgfältiger Pflege und der geplanten kurzen Liegezeit der Beckenzwinge relativ gering.

Die Beckenzwinge ist für die Notfallstabilisierung und nicht als Ausbehandlungsmaßnahme konzipiert.

\section{Fixateur externe}

Mit zum Teil sehr differenzierten Fixateur externe-,„Türmen“ hatte man in der Vergangenheit versucht, auch komplexe Instabilitäten des vorderen und hinteren Beckenrings zu beherrschen. Diese Ver- 
fahren sind zugunsten einfacherer Montagen verlassen worden. Während früher auch die Schanzschen Schrauben im Bereich des ventralen Beckenkamms eingebracht worden sind, hierbei aber häufiger eine rasche Lockerung und mangelnde Stabilität die Folge war, werden die Schanzschen Schrauben jetzt bevorzugt im so genannten supraazetabulären Beckenknochen verankert. Hierdurch hat man einen entscheidenden Gewinn an Effektivität verzeichnet.

Die Schanzschen Schrauben werden in Rückenlage des Patienten über kurzstreckige Inzisionen von ventral her implantiert. Hierbei ist zu beachten, dass bei weit klaffender Symphyse im Rahmen der Reposition ein zum Teil erheblicher Weg für die Schanzschen Schrauben in den Weichteilen zurückzulegen ist, deswegen müssen die Inzisionen eventuell nach medial vergrößert werden. Die Platzierung der Schanzschen Schrauben erfolgt nach Vorbohren mit dem 3,5mm-Bohrer unterhalb der Spina iliaca anterior superior. Die Verwendung von 5$\mathrm{mm}$-Schanzschen Schrauben ist die Regel, allerdings können auch bei entsprechender Knochenstruktur und kräftigem Körperbau Schrauben mit 6 mm Durchmesser verwendet werden. Sie suchen sich dann ihren Weg beim Eindrehen alleine nach dorsal innerhalb des Darmbeins. Für die Insertion ist ein Bildverstärker sinnvoll, um die Schrauben exakt platzieren zu können, zwei Schrauben auf jeder Seite sind die Regel (Abb.5).

Verbunden werden die Schanzschen Schrauben durch einfache Fixateur externe-Rohre oder auch Kohlefaserstangen, wobei die unterschiedlichsten Montagen mit oder ohne zwischengeschaltete Rohr-zu-Rohr-Backen möglich sind. Nach manueller Reposition des Beckens werden die Backen festgezogen. Die Verstrebung der einen Montage gegenüber der anderen ergibt eine noch größere Stabilität (Abb.6).

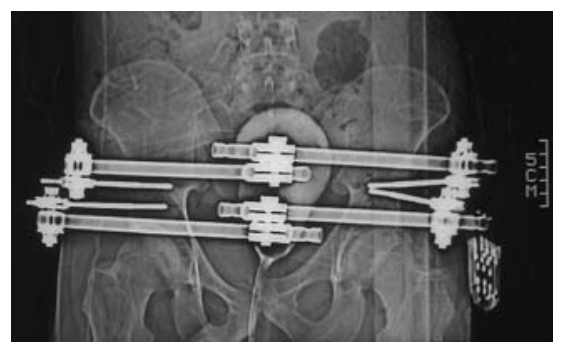

Abb. 5 Röntgenaufnahme des Beckens mit angelegtem Fixateur externe. Die Schanzschen Schrauben sind im supraazetabulären Knochen verankert.

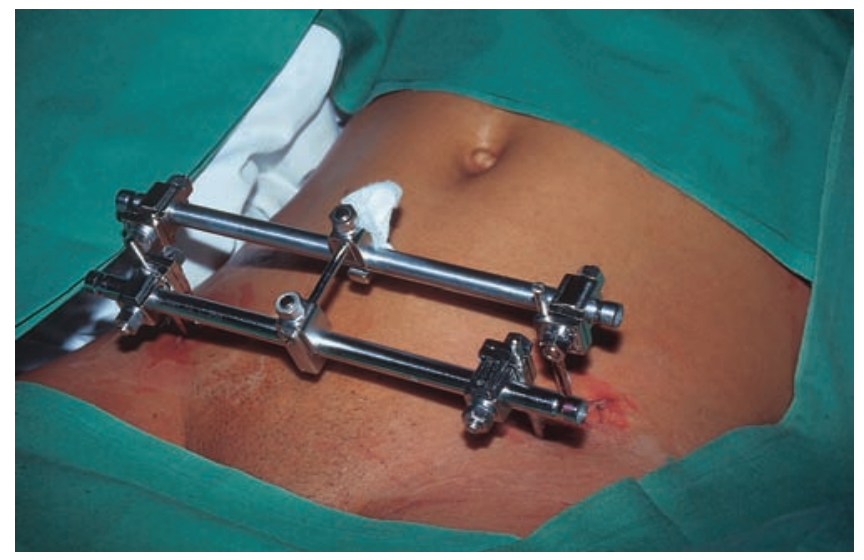

Abb. 6 Klinisches Beispiel für einen liegenden Beckenfixateur. Einfache ventrale Verstrebung ohne Zeltmontage.

Wegen der optimalen Stabilität ist eine Verankerung der Schanzschen Schrauben im supraazetabulären Knochen anzustreben.

Auf die hintere Beckenregion kann mit dem Fixateur externe nur wenig Einfluss genommen werden.

\section{Risiken}

Wie bei der Beckenzwinge können sich Weichteilkomplikationen an der Eintrittstelle der Schanzschen Schrauben und Probleme durch Fehlplatzierung ergeben:

Verletzung von A. und V. femoralis

- Verletzung des Hüftgelenks

- Verletzung des N. cutaneus femoris lateralis

- Lokale Weichteilprobleme an den Eintrittstellen

- Kompression von Bauchdecke/Abdomen

Kompression der Blase

Aufgrund der größeren Tiefe und der schmalen Knochenkontur ist die Platzierung der Schanzschen Schrauben unterhalb der Spina iliaca anterior superior schwieriger als ein Einbringen in den Darmbeinkamm. Sowohl der Bohrer wie auch die Schrauben können hier nach innen oder außen abgleiten. Dies bringt das Risiko einer Verletzung von Strukturen innerhalb der Beckenschaufel mit sich. Hierbei ist vor allem an das femorale Gefäßbündel zu denken. Die Verwendung eines Troikar-Buchsen-Systems bringt hier Vorteile, weil die Schanzsche Schraube unter dem Schutz der Buchse eingebracht werden kann und damit eine Verletzung von Weichteilen ausgeschlossen wird. Um eine Verletzung des Hüftgelenks zu vermeiden, ist die Verwendung eines Bildverstärkers ratsam.
Durch seine anatomischen Nachbarschaft ist vor allem der Nervus cutaneus femoris laterialis gefährdet, für den eine große interindividuelle Variation in Bezug auf seinen Verlauf bekannt ist. Sein Ausfall bedeutet eine lästige Gefühlsstörung auf der Oberschenkelvorderseite.

Der Verlauf der Schanzschen Schrauben leicht konvergierend sollte gerade bei der open-book-(Symphysenzerreißung) Verletzung bedacht werden. Je größer die Dislokation und damit je mehr Repositionsweg zu bewerkstelligen ist, desto exakter muss die Hautinzision platziert werden, um eine Weichteilirritation mit nachfolgendem Infekt $\mathrm{zu}$ vermeiden. Selbstverständlich sollte bei zu großer Weichteilspannung im Bereich der Insertionsstellen die Hautinzision nachkorrigiert werden. Regelmäßig kommt es bei Schwerverletzten zu dem Problem, dass bei zu wenig Abstand der ventralen Verbindungsstangen zum Abdomen durch eine in der Frühphase auftretende Darmatonie mit Aufblähung des Abdomens Kontakt zwischen den Verbindungsrohren und der vorderen Bauchwand entsteht. Dies erfordert nicht selten eine Nachkorrektur der Montage. Es sollte deshalb von vorne herein eine derartige mögliche Entwicklung bedacht und ein entsprechend großzügiger Abstand im Rahmen der Erstmontage eingeplant werden. Hierbei ist auch auf eine ausreichende Länge der Schanzschen Schrauben zu achten, damit die Fixationsbacken nicht Weichteilprobleme bedingen.

Eine seltene Komplikation besteht in der Quetschung der Blase zwischen den Schambeinästen, wenn eine Symphysenzerreißung mit dem Fixateur externe gedeckt reponiert wird. Deswegen ist hierbei schonendes Vorgehen im Rahmen der Reposition angezeigt. 


\section{Schlussfolgerung}

Für Beckenzwinge und Beckenfixateur ergeben sich klare Indikationen im Rahmen der Notfallbehandlung von instabilen Beckenverletzungen (Tscherne):

Mit der Beckenzwinge ist der instabile hintere Beckenring, mit dem Fixateur externe die ventrale Instabilität gut therapierbar. Die Effektivität beider Verfahren erfordert eine exakte Platzierung der Verankerungsschrauben, vor allem beim Fixateur externe empfiehlt sich hier eine Durchleuchtungskontrolle, um bei der empfohlenen supraazetabulären Implantation der Schanzschen Schrauben keine Verletzung des Hüftgelenkes zu riskieren. Bei Abweichung von den idealen Insertionspunkten besteht für beide Verfahren das Risiko einer Verletzung von benachbarten Gefäß- und Nervenstrukturen oder Instabilität der Montage. Trotz der anzustrebenden kurzen Liegezeiten dieser äußeren Stabilisierungsmittel muss von Anfang an eine konsequente Kontrolle und Pflege der Weichteile im Bereich der Eintrittsstellen erfolgen, um das Risiko von Weichteilkomplikationen gering zu halten.

\section{Literatur}

1 Tscherne H, Pohlemann T. (1998) Becken und Acetabulum. Berlin Heidelberg New York: Springer-Verlag

2 Pohlemann T, Gänsslen A, Hartung S. (1998) Beckenverletzungen/Pelvic injuries: Results of the German Multicenter Study Group. Berlin Heidelberg New York: Springer-Verlag

3 Ruedi TP, Murphy WM. (2003) AO Prinzipien des Frakturmanagements Stuttgart New York: Georg Thieme Verlag (ISBN 3-13129661-5)

\section{Prof. Dr. med. Franz Maurer}

Chefarzt der Abteilung für Unfall- und Wiederherstellungschirurgie, operative Orthopädie

Krankenhaus St. Elisabeth der Oberschwaben Klinik gGmbH, Ravensburg St. Elisabeth-Str. 15

D-88212 Ravensburg 